\title{
Dynamics and Stability of a Two Degree of Freedom Oscillator With an Elastic Stop
}

\author{
Madeleine Pascal \\ Laboratoire Systèmes Complexes, \\ Université d'Evry Val d'Essonne et CNRS FRE \\ 2494, 40 rue du Pelvoux, \\ 91020 Evry cedex, France \\ e-mail: mpascal@iup.univ-evry.fr
}

\begin{abstract}
A two degree of freedom oscillator with a colliding component is considered. The aim of the study is to investigate the dynamic behavior of the system when the stiffness obstacle changes to a finite value to an infinite one. Several cases are considered. First, in the case of rigid impact and without external excitation, a family of periodic solutions are found in analytical form. In the case of soft impact, with a finite time duration of the shock, and no external excitation, the existence of periodic solutions, with an arbitrary value of the period, is proved. Periodic motions are also obtained when the system is submitted to harmonic excitation, in both cases of rigid or soft impact. The stability of these periodic motions is investigated for these four cases.
\end{abstract}

Keywords: Nonlinear Vibrations, Two Degree of Freedom Oscillator, Rigid and Soft Impact, Periodic Motion, Stability, Forced and Unforced System

\section{Introduction}

Vibrating systems with clearance between the moving parts are frequently encountered in technical applications. These systems with impacts are strongly nonlinear; they are usually modeled as a spring-mass system with amplitude constraint. Such systems have been the subject of several investigations, mainly in the simplest case of a one degree of freedom system [1-4] and more seldom for multidegree of freedom systems [5-8]. On the other hand, the system behavior during the contact between the moving parts can be described as rigid impact, usually associated with a restitution coefficient, or modeled as soft impact, with a finite time duration of the shock. Several other parameters like damping, external excitation, influence the behavior of the system.

The work is the continuation of a previous paper [9], in which a two degree of freedom oscillator is considered. The nonlinearity in this case comes from the presence of two fixed stops limiting the motion of one mass. Assuming no damping and no external excitation, the behavior of the system is investigated when the obstacles stiffness changes from a finite value to an infinite one. In both cases, a family of symmetrical periodic solutions, with two impacts per period, is obtained in analytical form.

In the present paper, a two degree of freedom system in the presence of one fixed obstacle is considered. Assuming that no damping occurs, we investigate four cases: Unforced system with rigid impact, unforced system with soft impact, forced system (with harmonic excitation) with rigid impact and, at last, forced system with soft impact. In all cases, periodic solutions are found and stability results of these particular motions are obtained.

\section{Problem Formulation}

The system under consideration (Fig. 1) is a generalization of the double oscillator investigated in the paper [9]. It consists of two masses $m_{1}$ and $m_{2}$ connected by linear springs of stiffness $k_{1}$ and $k_{2}$. The displacement $z_{1}$ of the mass $m_{1}$ is limited by the presence of a fixed stop. When $z_{1}$ is greater than the clearance, a contact of the first mass with the stop occurs; this contact gives rise to a restoring force associated to a spring stiffness $k_{3}$. The mathematical model of the system is given by:

$$
M \ddot{z}+K z=F+P \cos (\omega t+\varphi), \quad z=\left(z_{1}, z_{2}\right)^{t}, \quad F=\left(\begin{array}{l}
f\left(z_{1}\right) \\
0
\end{array}\right),
$$

$$
\begin{aligned}
f\left(z_{1}\right)= & \left\{\begin{array}{ll}
-k_{3}\left(z_{1}-1\right) & z_{1}>1 \\
0 & z_{1}<1
\end{array}, \quad M=\left(\begin{array}{cc}
m_{1} & 0 \\
0 & m_{2}
\end{array}\right),\right. \\
& K=\left(\begin{array}{cc}
k_{1} & -k_{1} \\
-k_{1} & k_{1}+k_{2}
\end{array}\right)
\end{aligned}
$$

$P=\left(\begin{array}{l}P_{1} \\ P_{2}\end{array}\right)$ and $\varphi$ are the amplitude and the phase angle of the harmonic excitation.

\section{Unforced System}

Let us consider the system without external excitation $(P=0)$.

3.1 Rigid Impact. When the stiffness obstacle $k_{3}$ tends to infinity, a rigid impact of the first mass against the stop occurs. Starting from initial positions $z_{0}=\left(\begin{array}{l}1 \\ y\end{array}\right)$ and initial velocities $\dot{z}_{0}$ $=\left(\begin{array}{l}u \\ w\end{array}\right)(u>0)$, corresponding to a contact of the first mass against the stop, assuming a perfect elastic impact, the new positions $z_{c}$ and the new velocities $\dot{z}_{c}$ after the shock are obtained by:

$$
\left(\begin{array}{c}
z_{c} \\
\dot{z}_{c}
\end{array}\right)=\left(\begin{array}{cc}
I & 0 \\
0 & E
\end{array}\right)\left(\begin{array}{c}
z_{0} \\
\dot{z}_{0}
\end{array}\right), \quad I=\left(\begin{array}{cc}
1 & 0 \\
0 & 1
\end{array}\right), \quad E=\left(\begin{array}{cc}
-1 & 0 \\
0 & 1
\end{array}\right)
$$

After the impact, the system performs a free motion defined by:

$$
\begin{aligned}
\left(\begin{array}{l}
z \\
\dot{z}
\end{array}\right)= & C(t)\left(\begin{array}{l}
z_{c} \\
\dot{z}_{c}
\end{array}\right), \quad C(t)=\left(\begin{array}{ll}
\Gamma_{1}(t) & \Gamma_{2}(t) \\
\Gamma_{3}(t) & \Gamma_{1}(t)
\end{array}\right), \quad \Gamma_{i}=\Lambda B_{i}(t) \Lambda^{-1} \\
& (i=1,2,3)
\end{aligned}
$$




$$
\begin{gathered}
\Lambda=\left(\begin{array}{cc}
1 & 1 \\
\lambda_{1} & \lambda_{2}
\end{array}\right), \quad B_{1}(t)=\left(\begin{array}{ll}
C_{1}(t) & 0 \\
0 & C_{2}(t)
\end{array}\right), \\
B_{2}(t)=\left(\begin{array}{cc}
\frac{S_{1}(t)}{\omega_{1}} & 0 \\
0 & \frac{S_{2}(t)}{\omega_{2}}
\end{array}\right),
\end{gathered}
$$

$B_{3}(t)=\dot{B}_{1}(t), C_{i}(t)=\cos \omega_{i} t, S_{i}(t)=\sin \omega_{i} t(i=1,2)$.

In these formulas, $\left(\omega_{1}, \omega_{2}\right)$ are the roots of the characteristic equation: $\Delta\left(\omega^{2}\right) \equiv \operatorname{det}\left(K-M \omega^{2}\right)=0$ while $\Psi_{i}=\left(\begin{array}{c}1 \\ \lambda_{i}\end{array}\right)$ are defined by $\left(K-M \omega_{i}^{2}\right) \Psi_{i}=0(i=1,2)$.

The following properties for the $\Gamma_{i}$ matrices hold:

$$
\Gamma_{1}^{2}(t)-\Gamma_{2}(t) \Gamma_{3}(t)=I, \quad \Gamma_{i}(t) \Gamma_{j}(t)=\Gamma_{j}(t) \Gamma_{i}(t) \text { for } i, j=1,2,3
$$

Moreover, the coefficients $C_{i j}(t)$ of the 4 by 4 matrix $C(t)$ satisfy the property:

$$
C_{i j}(t)=C_{i-2, j-2}(t), \quad(i, j=3,4)
$$

Let us investigate if for a set of initial conditions $Z_{0}=\left(\begin{array}{l}z_{0} \\ \dot{z}_{0}\end{array}\right), z_{0}$ $=\left(\begin{array}{l}1 \\ y\end{array}\right), \dot{z}_{0}=\left(\begin{array}{c}u>0 \\ w\end{array}\right)$, it is possible to obtain a periodic solution of period $T$, with one impact per period.

The free motion performed by the system after the rigid impact finishes at time $t=T$ when $z_{1}(T)=1$ and $\dot{z}_{1}(T)>0$. Let us denote by $Z_{f}=\left(\begin{array}{l}z_{f} \\ i_{f}\end{array}\right)$ the positions and the velocities reached by the system at $t=T$.

The condition to obtain such a periodic motion is given by:

$$
Z_{f} \equiv C(T) Z_{c}=Z_{0}, \quad Z_{c}=H_{0} Z_{0}, \quad H_{0}=\left(\begin{array}{cc}
I & 0 \\
0 & E
\end{array}\right)
$$

Let us introduce the position $Z_{s}$ reached by the system from the initial position $Z_{0}$ after a backward motion of duration $T: Z_{s}=C$ $(-T) Z_{0}$. The condition (7) is equivalent to: $Z_{s}=Z_{c}$.

It results for the determination of the four scalar parameters $(y, u, w, T)$ the four scalar equations:

$$
\left(-H_{0}+C(-T)\right) Z_{0}=0, \quad Z_{0}=(1, y, u, w)^{t}
$$

or equivalently:

$$
\begin{gathered}
\left(\Gamma_{1}-I\right) z_{0}-\Gamma_{2} \dot{z}_{0}=0 \\
-\Gamma_{3} z_{0}+\left(\Gamma_{1}-E\right) \dot{z}_{0}=0
\end{gathered} \Gamma_{i}=\Gamma_{i}(T) \quad(i=1,2,3)
$$

Taking into account the properties (5) of the $\Gamma_{i}$ matrices, the system (8) leads to:

$$
\begin{gathered}
z_{0}=\left(\Gamma_{1}-I\right)^{-1} \Gamma_{2} \dot{z}_{0} \\
(E+I) \dot{z}_{0}=0
\end{gathered}
$$

The last equation of (9) reduces to $w=0$. From the first one, $y$ and $u$ are obtained in terms of the period:

$$
\begin{gathered}
y=\frac{\left(\omega_{2} t_{2}-\omega_{1} t_{1}\right) \lambda_{1} \lambda_{2}}{\lambda_{2} \omega_{2} t_{2}-\lambda_{1} \omega_{1} t_{1}}, \quad u=\frac{\left(\lambda_{1}-\lambda_{2}\right) \omega_{1} \omega_{2} t_{1} t_{2}}{\lambda_{2} \omega_{2} t_{2}-\lambda_{1} \omega_{1} t_{1}}, \\
t_{i}=\tan \left(\frac{\omega_{i} T}{2}\right) \quad(i=1,2)
\end{gathered}
$$

In case of rigid impact, a family of periodic solutions is obtained for which the initial conditions are defined in terms of the period and the initial velocity $w$ of the nonimpacting mass is zero. For these particular motions, the conditions giving the positions and the velocities after the shock can be formulated by:

$$
z_{c}=z_{0}, \quad \dot{z}_{c}=-\dot{z}_{0}
$$

These results are similar to the results obtained in Ref. [9]. The system considered in this previous paper is a symmetrical system with respect to the position $z_{1}$ of the first mass and it can be

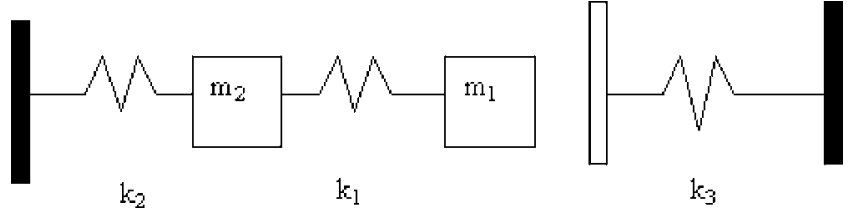

Fig. 1 Double oscillator

expected that the obtained results are due to this property. But it is not the real explanation because the system investigated now is not symmetrical.

3.2 Soft Impact. Let us assume that the stiffness obstacle is bounded. The mathematical model of the system is given by: For $z_{1}<1$

$$
M \ddot{z}+K z=0
$$

For $z_{1}>1$

$$
M \ddot{z}+K_{1} z=K_{3} \quad K_{1}=\left(\begin{array}{cc}
k_{1}+k_{3} & -k_{1} \\
-k_{1} & k_{1}+k_{2}
\end{array}\right) \quad K_{3}=\left(\begin{array}{c}
k_{3} \\
0
\end{array}\right)
$$

Let us assume that the initial conditions are given by: $Z_{0}$ $=(1, y, u, w)^{t} u>0$.

A periodic solution is defined in two steps:

- For $0 \leqslant t \leqslant \tau, z_{1}>1$, the system is defined by the motion equations (13). The time duration $\tau$ of this constraint motion is defined by the condition:

$$
z_{1}(\tau)=1
$$

Let us denote by $Z_{c}=Z(\tau)=\left(1, y_{c}, u_{c}, w_{c}\right)^{t}$ the value of the parameters at the end of shock, with the condition $u_{C}<0$.

- For $\tau \leqslant t \leqslant \tau+T$, a free motion obtained from Eqs. (12) and initial conditions $Z_{c}$ occurs. This motion finishes when $z_{1}(\tau$ $+T)=1$. Let us denote by $Z_{f}=Z(\tau+T)=\left(1, y_{f}, u_{f}, w_{f}\right)^{t}$ the value of the parameters at the end of free motion $\left(u_{f}>0\right)$. The condition to obtain a periodic orbit of period $\tau+T$ is given by

$$
Z_{f}=Z_{0}
$$

The piecewise linear systems (12) and (13) give the two parts of the motion in analytical form.

- For $0 \leqslant t \leqslant \tau$, the constraint motion is deduced from a modal analysis of system (13):

$$
\begin{aligned}
& Z(t)=H(t)\left(Z_{0}-d\right)+d, \quad d=\left(d_{1}, d_{2}, 0,0\right)^{t} \\
& d_{1}=\frac{k_{3}\left(k_{1}+k_{2}\right)}{k_{1} k_{2}+k_{3}\left(k_{1}+k_{3}\right)}, \quad d_{2}=\frac{k_{3} k_{1}}{k_{1} k_{2}+k_{3}\left(k_{1}+k_{2}\right)} \\
& H(t)=\left(\begin{array}{ll}
H_{1}(t) & H_{2}(t) \\
H_{3}(t) & H_{1}(t)
\end{array}\right), \quad H_{i}(t)=\Sigma G_{i}(t) \Sigma^{-1} \\
& (i=1,2,3), \quad \Sigma=\left(\begin{array}{cc}
1 & \mu_{2} \\
\mu_{1} & 1
\end{array}\right) \\
& G_{1}(t)=\left(\begin{array}{cc}
c_{1}(t) & 0 \\
0 & c_{2}(t)
\end{array}\right), \quad G_{2}=\left(\begin{array}{cc}
\frac{s_{1}(t)}{\sigma_{1}} & 0 \\
0 & \frac{s_{2}(t)}{\sigma_{2}}
\end{array}\right), \quad G_{3}=\dot{G}_{1}, \\
& c_{i}(t)=\cos \left(\sigma_{i} t\right), \quad s_{i}(t)=\sin \left(\sigma_{i} t\right), \quad(i=1,2)
\end{aligned}
$$

In these formulas, $\sigma_{1}, \sigma_{2}, \Phi_{1}=\left(\begin{array}{c}1 \\ \mu_{1}\end{array}\right), \Phi_{2}=\left(\begin{array}{c}\mu_{2} \\ 1\end{array}\right)$ define the characteristic frequencies and the eigenvectors of the constraint 
system (13). For the $H_{i}$ matrices, the properties (5) obtained for the $\Gamma_{i}$ matrices hold, together with the property $H_{i j}(t)$ $=H_{i-2, j-2}(t),(i, j=3,4)$ for the coefficients $H_{i j}(t)$ of the matrix $\mathrm{H}(\mathrm{t})$.

- For $\tau \leqslant t \leqslant \tau+T$, the free motion is obtained from $Z(t)=C(t$ $-\tau) Z_{c}$ where the matrix $C$ is defined by formulas (3) and (4) and $Z_{c}=H(\tau)\left(Z_{0}-d\right)+d$.

Let us introduce the positions and the velocities $Z_{s}$ $=\left(z_{1 s}, z_{2 s}, \dot{z}_{1 s}, \dot{z}_{2 s}\right)^{t}$ of the system after a backward motion of duration $T$ from the initial position $Z_{0}$. The condition (15) of periodicity is reformulated as

$$
\begin{gathered}
Z_{s} \equiv C(-T) Z_{0}=Z_{c} \\
Z_{c}=\left(\begin{array}{c}
z_{c} \\
\dot{z}_{c}
\end{array}\right)=\left(\begin{array}{c}
H_{1}\left(z_{0}-d_{0}\right)+H_{2} \dot{z}_{0}+d_{0} \\
H_{3}\left(z_{0}-d_{0}\right)+H_{1} \dot{z}_{0}
\end{array}\right), \quad d_{0}=\left(d_{1}, d_{2}\right)^{t} \\
Z_{s}=\left(\begin{array}{c}
z_{s} \\
\dot{z}_{s}
\end{array}\right)=\left(\begin{array}{c}
\Gamma_{1} z_{0}-\Gamma_{2} \dot{z}_{0} \\
-\Gamma_{3} z_{0}+\Gamma_{1} \dot{z}_{0}
\end{array}\right), \quad H_{i}=H_{i}(\tau), \quad \Gamma_{i}=\Gamma_{i}(T) \\
(i=1,2,3)
\end{gathered}
$$

The condition (19) is equivalent to

$$
\begin{aligned}
& X_{1}=X_{2}, \quad Y_{1}=Y_{2} \\
& X_{1} \equiv z_{c}-z_{0}=\left(H_{1}-I\right)\left(z_{0}-d_{0}\right)+H_{2} \dot{z}_{0}, \quad X_{2} \equiv z_{s}-z_{0}=\left(\Gamma_{1}-I\right) z_{0} \\
& -\Gamma_{2} \dot{z}_{0} \\
& Y_{1} \equiv \dot{z}_{c}+\dot{z}_{0}=H_{3}\left(z_{0}-d_{0}\right)+\left(H_{1}+I\right) \dot{z}_{0}, \quad Y_{2} \equiv \dot{z}_{s}+\dot{z}_{0}=-\Gamma_{3} z_{0} \\
& \quad+\left(\Gamma_{1}+I\right) \dot{z}_{0}
\end{aligned}
$$

From the properties (5) of $\Gamma_{i}$ and $H_{i}$, we deduce:

$$
\begin{aligned}
& Y_{i}=P_{i} X_{i}(i=1,2) \\
& P_{1}=H_{2}^{-1}\left(H_{1}+I\right), \quad P_{2}=-\Gamma_{2}^{-1}\left(\Gamma_{1}+I\right)
\end{aligned}
$$

The condition (21) leads to

$$
X_{1}=X_{2}, \quad P_{1} X_{1}=P_{2} X_{2}
$$

Two possible cases of periodic solutions can be deduced from (24), namely:

$$
X_{1}=X_{2}=0 \quad \text { or } X_{1}=X_{2}, \quad \operatorname{det}\left(P_{1}-P_{2}\right)=0
$$

3.3 Existence of Periodic Motions (Soft Impact). Let us discuss the first conditions (25). In this case, from (22), we deduce:

$$
z_{c}=z_{0}, \quad \dot{z}_{c}=-\dot{z}_{0}
$$

The condition (14) is fulfilled and the initial conditions are obtained from the equations:

$$
\begin{gathered}
\left(H_{1}-I\right)\left(z_{0}-d_{0}\right)+H_{2} \dot{z}_{0}=0 \\
\left(\Gamma_{1}-I\right) z_{0}-\Gamma_{2} \dot{z}_{0}=0
\end{gathered}
$$

This system provides four scalar equations for the determination of the five parameters $(y, u, w, \tau, T)$. It results that, as in the case of rigid impact, $T$ and hence the period can be chosen arbitrarily. Moreover, the conditions (11) and (26) obtained at the end of the shock are the same for both rigid and soft impacts. From (27), we deduce:

$$
\begin{gathered}
\dot{z}_{0}=-H_{2}^{-1}\left(H_{1}-I\right)\left(z_{0}-d_{0}\right) \\
{\left[\Gamma_{1}-I+\Gamma_{2} H_{2}^{-1}\left(H_{1}-I\right)\right] z_{0}=\Gamma_{2} H_{2}^{-1}\left(H_{1}-I\right) d_{0}}
\end{gathered}
$$

The last equation (28), after the elimination of $y$, provides a relation $F(\tau, T)=0$ between the time duration $\tau$ of the shock and the time duration $T$ of the free motion.

-The other case $X_{1}=X_{2}, \operatorname{det}\left(P_{1}-P_{2}\right)=0$ leads to no solution (see Appendix A).

In both cases (soft or rigid impact), a family of periodic motions is obtained, with an arbitrary value of the period. Moreover, the conditions (26) obtained at the end of the shock for soft impact are consistent with Newton rules of rigid impact (11) with a restitution coefficient equal to one, i.e., with assumption of ideal elastic impact. This rather remarkable result has already been obtained for the symmetrical system of Ref. [9].

\section{Forced System}

Let us assume that the two masses are subjected to harmonic external excitations of period $2 \pi / \omega$, constant amplitudes $P_{1}, P_{2}$ and constant phase angle $\varphi$. From the results obtained in the previous paragraph, where a family of periodic orbits is found with an arbitrary value of the period, it can be expected that for the forced system, periodic solutions of period $2 \pi / \omega$ exist.

4.1 Rigid Impact. Let us investigate the case of rigid impact. Starting from the initial conditions $Z_{0}=(1, y, u, w)^{t}(u>0)$, the conditions $Z_{c}=\left(1, y_{c}, u_{c}, w_{c}\right)^{t}$ after the shock are obtained from (2) and the free motion performed by the system is given by:

$$
\begin{gathered}
z=\Gamma_{1}(t)\left(z_{0}-R \cos \varphi\right)+\Gamma_{2}(t)\left(\dot{z}_{c}+R \omega \sin \varphi\right)+R \cos (\omega t+\varphi) \\
\dot{z}=\Gamma_{3}(t)\left(z_{0}-R \cos \varphi\right)+\Gamma_{1}(t)\left(\dot{z}_{c}+R \omega \sin \varphi\right)-R \omega \sin (\omega t+\varphi)
\end{gathered}
$$

where $R=\left(R_{1}, R_{2}\right)^{t}$ is the amplitude of the response defined by:

$$
\begin{gathered}
R_{1}=A_{1}+A_{2}, \quad R_{2}=\lambda_{1} A_{1}+\lambda_{2} A_{2} \\
A_{i}=\frac{P_{1}+\lambda_{i} P_{2}}{\left(\omega_{i}^{2}-\omega^{2}\right)\left(m_{1}+\lambda_{i}^{2} m_{2}\right)}, \quad(i=1,2)
\end{gathered}
$$

The free motion finishes at time $t=T$ when $z_{1}(T)=1, \dot{z}_{1}(T)>0$. Let us denote by $Z_{f}=\left(z_{f}, \dot{z}_{f}\right)^{t}$ the positions and the velocities reached by the system at this time. The condition to obtain a periodic motion of period $T$ is:

or :

$$
Z_{f}=Z_{0}
$$

$$
\begin{gathered}
z_{0}=\Gamma_{1}(T)\left(z_{0}-R \cos \varphi\right)+\Gamma_{2}(T)\left(\dot{z}_{c}+R \omega \sin \varphi\right)+R \cos (\omega T+\varphi) \\
\dot{z}_{0}=\Gamma_{3}(T)\left(z_{0}-R \cos \varphi\right)+\Gamma_{1}(T)\left(\dot{z}_{c}+R \omega \sin \varphi\right)-R \omega \sin (\omega T+\varphi) \\
\dot{z}_{c}=E \dot{z}_{0}
\end{gathered}
$$

Let us assume that $T=2 \pi / \omega, \varphi=0, \dot{z}_{c}=-\dot{z}_{0}$. A periodic motion of period $2 \pi / \omega$ is obtained if the initial conditions $Z_{0}=(1, y, u, 0)^{t}$ are defined by the system:

$$
\begin{gathered}
\left(\Gamma_{1}-I\right)\left(z_{0}-R\right)-\Gamma_{2} \dot{z}_{0}=0 \\
\Gamma_{3}\left(z_{0}-R\right)-\left(\Gamma_{1}+I\right) \dot{z}_{0}=0 \\
\Gamma_{i}=\Gamma_{i}(2 \pi / \omega), \quad(i=1,2,3)
\end{gathered}
$$

Taking into account the properties (5) of the $\Gamma_{i}$ matrices, this system reduces to

$$
\dot{z}_{0}=\Gamma_{2}^{-1}\left(\Gamma_{1}-I\right)\left(z_{0}-R\right)
$$

and the corresponding values of $y$ and $u$ are obtained:

$$
\begin{gathered}
y=R_{2}+\frac{\left(\omega_{2} t_{2}-\omega_{1} t_{1}\right) \lambda_{1} \lambda_{2}}{\lambda_{2} \omega_{2} t_{2}-\lambda_{1} \omega_{1} t_{1}}\left(1-R_{1}\right), \\
u=\left(1-R_{1}\right) \frac{\left(\lambda_{1}-\lambda_{2}\right) \omega_{1} \omega_{2} t_{1} t_{2}}{\lambda_{2} \omega_{2} t_{2}-\lambda_{1} \omega_{1} t_{1}}, \quad t_{i}=\tan \left(\frac{\omega_{i} \pi}{\omega}\right) \quad(i=1,2)
\end{gathered}
$$

Remark: In more general cases, the impact is described by a 
restitution coefficient $r(0<r<1)$. The initial conditions and the phase angle related to a periodic solution of period $2 \pi / \omega$ can also be obtained in analytical form [10]. A similar solution has been studied in paper [6].

4.2 Soft Impact. When the stiffness obstacle is bounded, the motion equations of the system are given by:

$$
\begin{gathered}
M \ddot{z}+K_{1} z=K_{3}+P \cos (\omega t+\varphi) \quad z_{1}>1 \\
M \ddot{z}+K z=P \cos (\omega t+\varphi) \quad z_{1}<1
\end{gathered}
$$

From the initial condition $Z_{0}=(1, y, u, w)^{t}(u>0)$, the solution is defined in two parts:

- For $0<t<\tau, z_{1}>1$, the solution is given by:

$$
\begin{aligned}
z= & H_{1}(t)\left(z_{0}-d_{0}-Q \cos \varphi\right)+H_{2}(t)\left(\dot{z}_{0}+Q \omega \sin \varphi\right)+d_{0} \\
& +Q \cos (\omega t+\varphi) \\
\dot{z}= & H_{3}(t)\left(z_{0}-d_{0}-Q \cos \varphi\right)+H_{1}(t)\left(\dot{z}_{0}+Q \omega \sin \varphi\right) \\
& -Q \omega \sin (\omega t+\varphi)
\end{aligned}
$$

$Q=\left(Q_{1}, Q_{2}\right)^{t}$ is the response amplitude defined by:

$$
\begin{gathered}
Q_{1}=\frac{P_{1}\left(1+\mu_{2}^{2}\right)+P_{2}\left(\mu_{1}+\mu_{2}\right)}{\left(\sigma_{1}^{2}-\omega^{2}\right)\left(m_{1}+m_{2} \mu_{1}^{2}\right)}, \\
Q_{2}=\frac{P_{1}\left(\mu_{1}+\mu_{2}\right)+P_{2}\left(1+\mu_{1}^{2}\right)}{\left(\sigma_{2}^{2}-\omega^{2}\right)\left(m_{2}+m_{1} \mu_{2}^{2}\right)}
\end{gathered}
$$

The time duration $\tau$ of this motion is obtained from the condition $z_{1}(\tau)=1$. Let us denote $Z_{c}=\left(1, y_{c}, u_{c}, w_{c}\right)^{t}$ the value of the parameters at $t=\tau\left(u_{c}<0\right)$.

- For $\tau<t<\tau+T$, the motion of the system is defined by:

$$
\begin{aligned}
z= & \Gamma_{1}(t-\tau)\left(z_{c}-R \cos \psi\right)+\Gamma_{2}(t-\tau)\left(\dot{z}_{c}+R \omega \sin \psi\right) \\
& +R \cos (\omega t+\varphi) \\
\dot{z}= & \Gamma_{3}(t-\tau)\left(z_{c}-R \cos \psi\right)+\Gamma_{1}(t-\tau)\left(\dot{z}_{c}+R \omega \sin \psi\right) \\
& -R \omega \sin (\omega t+\varphi)
\end{aligned}
$$

where $R=\left(R_{1}, R_{2}\right)^{t}$ is defined by (30) and $\psi=\omega \tau+\varphi$. This motion finishes at time $t=\tau+T$ when $z_{1}(\tau+T)=1, \dot{z}_{1}(\tau+T)$ $>0$. If $Z_{f}=\left(z_{f}, \dot{z}_{f}\right)^{t}$ denote the positions and the velocities reached by the system at this time, the condition to obtain a periodic motion of period $\tau+T$ is $Z_{f}=Z_{0}$. Let us assume that $\tau+T=2 \pi / \omega, \varphi=-\omega \tau / 2$. At the end of the first part of the motion $(t=\tau)$, the positions and the velocities are given by:

$$
\begin{gathered}
z_{c}=H_{1}\left(z_{0}-d_{0}-Q \cos \widetilde{\varphi}_{0}\right)+H_{2}\left(\dot{z}_{0}-Q \omega \sin \widetilde{\varphi}_{0}\right)+d_{0} \\
+Q \cos \widetilde{\varphi}_{0} \\
\dot{z}_{c}=H_{3}\left(z_{0}-d_{0}-Q \cos \widetilde{\varphi}_{0}\right)+H_{1}\left(\dot{z}_{0}-Q \omega \sin \widetilde{\varphi}_{0}\right)-Q \omega \sin \widetilde{\varphi}_{0} \\
\widetilde{\varphi}_{0}=\omega \tau / 2, \quad H_{i}=H_{i}(\tau) \quad(i=1,2,3)
\end{gathered}
$$

At the end of the second part of the motion $(t=2 \pi / \omega)$, we obtain:

$$
\begin{gathered}
z_{f}=\Gamma_{1}\left(z_{c}-R \cos \tilde{\varphi}_{0}\right)+\Gamma_{2}\left(\dot{z}_{c}+R \omega \sin \tilde{\varphi}_{0}\right)+R \cos \tilde{\varphi}_{0} \\
\dot{z}_{f}=\Gamma_{3}\left(z_{c}-R \cos \widetilde{\varphi}_{0}\right)+\Gamma_{1}\left(\dot{z}_{c}+R \omega \sin \widetilde{\varphi}_{0}\right)+R \omega \sin \widetilde{\varphi}_{0} \\
\Gamma_{i}=\Gamma_{i}(2 \pi / \omega-\tau) \quad(i=1,2,3)
\end{gathered}
$$

The conditions of periodicity are reformulated as:

$$
\tilde{X}_{1}=\tilde{X}_{2}, \quad \tilde{Y}_{1}=\tilde{Y}_{2}
$$

$$
\begin{gathered}
\tilde{X}_{1} \equiv z_{c}-z_{0}=\left(H_{1}-I\right)\left(z_{0}-d_{0}-Q \cos \widetilde{\varphi}_{0}\right) \\
+H_{2}\left(\dot{z}_{0}-Q \omega \sin \widetilde{\varphi}_{0}\right) \\
\tilde{Y}_{1} \equiv \dot{z}_{c}+\dot{z}_{0}=H_{3}\left(z_{0}-d_{0}-Q \cos \widetilde{\varphi}_{0}\right) \\
+\left(H_{1}+I\right)\left(\dot{z}_{0}-Q \omega \sin \widetilde{\varphi}_{0}\right) \\
\tilde{X}_{2} \equiv z_{s}-z_{0}=\left(\Gamma_{1}-I\right)\left(z_{0}-R \cos \widetilde{\varphi}_{0}\right)-\Gamma_{2}\left(\dot{z}_{0}-R \omega \sin \widetilde{\varphi}_{0}\right) \\
\tilde{Y}_{2} \equiv \dot{z}_{s}+\dot{z}_{0}=-\Gamma_{3}\left(z_{0}-R \cos \widetilde{\varphi}_{0}\right)+\left(\Gamma_{1}+I\right)\left(\dot{z}_{0}-R \omega \sin \widetilde{\varphi}_{0}\right) \\
Z_{s}=\left(z_{s}, \dot{z}_{s}\right)^{t}=C(-T) Z_{0}
\end{gathered}
$$

As in the case of an unforced system taking into account the properties of the $H_{i}$ and $\Gamma_{i}$ matrices, the solution of system (41) is given by:

$$
\tilde{X}_{1}=\tilde{X}_{2}=0, \quad \tilde{Y}_{1}=\tilde{Y}_{2}=0
$$

We deduce for the forced system the existence of a periodic motion of period $2 \pi / \omega$ for which the conditions at the end of the shock are $z_{c}=z_{0}, \dot{z}_{c}=-\dot{z}_{0}$. The time duration $\tau$ of the shock and the initial conditions $(y, u, w)$ are obtained from the first part of system (44):

$$
\begin{gathered}
\dot{z}_{0}=Q \omega \sin \widetilde{\varphi}_{0}-H_{2}^{-1}\left(H_{1}-I\right)\left(z_{0}-d_{0}-Q \cos \widetilde{\varphi}_{0}\right) \\
{\left[\Gamma_{2}^{-1}\left(\Gamma_{1}-I\right)+H_{2}^{-1}\left(H_{1}-I\right)\right] z_{0}=(Q-R) \omega \sin \widetilde{\varphi}_{0}} \\
\quad+\Gamma_{2}^{-1}\left(\Gamma_{1}-I\right) R \cos \widetilde{\varphi}_{0}+H_{2}^{-1}\left(H_{1}-I\right)\left(d_{0}+Q \cos \widetilde{\varphi}_{0}\right)
\end{gathered}
$$

These formulas give the relations (28) when $Q=R=0$ (unforced system).

\section{Stability of Periodic Motions (Rigid Impact)}

5.1 Unforced System. Let us consider a periodic motion of period $T$ related to initial conditions $z_{00}=\left(\begin{array}{c}1 \\ y_{0}\end{array}\right), \dot{z}_{00}=\left(\begin{array}{c}u_{0} \\ 0\end{array}\right)$, where $z_{00}$, $\dot{z}_{00}$ are defined by:

$$
\begin{gathered}
\left(\Gamma_{1}-I\right) z_{00}-\Gamma_{2} \dot{z}_{00}=0 \quad \Gamma_{i}=\Gamma_{i}(T) \\
-\Gamma_{3} z_{00}+\left(\Gamma_{1}-E\right) \dot{z}_{00}=0
\end{gathered}
$$

Let us consider the perturbed motion defined by a set of new initial conditions

$$
\begin{aligned}
& z_{0}=z_{00}+d z_{0} \\
& \dot{z}_{0}=\dot{z}_{00}+d \dot{z}_{0}
\end{aligned} \text { where } d z_{0}=\left(\begin{array}{l}
0 \\
y
\end{array}\right), \quad d \dot{z}_{0}=\left(\begin{array}{l}
u \\
w
\end{array}\right)
$$

This motion is defined for $\mathrm{t}>0$, by:

$$
\begin{aligned}
& z=\Gamma_{1}(t) z_{0}+\Gamma_{2}(t) E \dot{z}_{0} \\
& \dot{z}=\Gamma_{3}(t) z_{0}+\Gamma_{1}(t) E \dot{z}_{0}
\end{aligned}
$$

This motion ends at $t=T+d T$, when $z_{1}(T+d T)=1$ and $\dot{z}_{1}(T+d T)$ $>0$. Let us denote by $z_{f}, \dot{z}_{f}$ the positions and the velocities of the system at this time.

$$
\begin{aligned}
& z_{f}=\Gamma_{1}(T+d T) z_{0}+\Gamma_{2}(T+d T) E \dot{z}_{0} \\
& \dot{z}_{f}=\Gamma_{3}(T+d T) z_{0}+\Gamma_{1}(T+d T) E \dot{z}_{0}
\end{aligned}
$$

Assuming small perturbations $d z_{0}, d \dot{z}_{0}$ of the initial conditions,

$$
\begin{gathered}
d z_{f}=z_{f}-z_{00}=\Gamma_{1} d z_{0}+\Gamma_{2} E d \dot{z}_{0}+L_{1} d T \\
d \dot{z}_{f}=\dot{z}_{f}-\dot{z}_{00}=\Gamma_{3} d z_{0}+\Gamma_{1} E d \dot{z}_{0}+L_{2} d T \\
L_{1}=\dot{\Gamma}_{1} z_{00}+\dot{\Gamma}_{2} E \dot{z}_{00}
\end{gathered}
$$




$$
\begin{gathered}
L_{2}=\dot{\Gamma}_{3} z_{00}+\dot{\Gamma}_{1} E \dot{z}_{00}, \quad d z_{f}=\left(\begin{array}{c}
0 \\
y_{f}
\end{array}\right), \quad d \dot{z}_{f}=\left(\begin{array}{c}
u_{f} \\
w_{f}
\end{array}\right) \\
\dot{\Gamma}_{i}=\dot{\Gamma}_{i}(T)
\end{gathered}
$$

The relations $\dot{\Gamma}_{1}=\Gamma_{3}, \dot{\Gamma}_{2}=\Gamma_{1}, \dot{\Gamma}_{3}=\Gamma_{2}^{-1} \Gamma_{1} \Gamma_{3}, E \dot{z}_{00}=-\dot{z}_{00}$ and (46) leads to the relations:

$$
L_{1}=\dot{z}_{00}, \quad L_{2}=\Gamma_{2}^{-1}\left(\Gamma_{1}+I\right) \dot{z}_{00}
$$

From (48), after the elimination of $d T$, we deduce:

$$
\begin{gathered}
y_{f}=C_{22} y-C_{23} u+C_{24} w \\
C_{12} y_{f}-C_{13} u_{f}-C_{14} w_{f}=C_{12} y-C_{13} u+C_{14} w \\
\left(C_{22}+1\right) y_{f}-C_{23} u_{f}-C_{24} w_{f}=\left(C_{22}+1\right) y-C_{23} u+C_{24} w
\end{gathered}
$$

or $A X_{f}=B X, X_{f}={ }^{t}\left(y_{f}, u_{f}, w_{f}\right), X={ }^{t}(y, u, w)$

$$
A=\left(\begin{array}{ccc}
1 & 0 & 0 \\
C_{22} & -C_{23} & -C_{24} \\
C_{12} & -C_{13} & -C_{14}
\end{array}\right), \quad B=\left(\begin{array}{ccc}
C_{22} & -C_{23} & C_{24} \\
1 & 0 & 0 \\
C_{12} & -C_{13} & C_{14}
\end{array}\right)
$$

The stability of the periodic impact solution is determined by the eigenvalues of the matrix $A^{-1} B$. If all the eigenvalues are inside the unit circle, the periodic solution is stable. If one of them is outside the unit circle, the solution is unstable. Critical cases occur if some eigenvalues lie on the unit circle, the other ones being strictly inside this circle.

Let us introduce the characteristic polynomial $P(\lambda)$ of the matrix $A^{-1} B$ :

$$
P(\lambda) \equiv \operatorname{det}\left(A^{-1} B-\lambda I_{3}\right)=-\left(\lambda^{3}+b_{2} \lambda^{2}+b_{1} \lambda+b_{0}\right)
$$

$\left(I_{3}\right.$ unitarian matrix of order 3$)$

$P(\lambda)=0$ is equivalent to $D(\lambda)=0, \quad D(\lambda) \equiv \operatorname{det}(B-\lambda A)$

From the properties: $D(1) \equiv \operatorname{det}(B-A)=0, \operatorname{det}(A)=\operatorname{det}(B)$, we $\operatorname{de}$ duce that one eigenvalue of $A^{-1} B$ is 1 and $b_{0} \equiv-\operatorname{det}\left(A^{-1} B\right)=-1$. The characteristic polynomial of $A^{-1} B$ takes the following form:

$$
P(\lambda)=(1-\lambda)\left[\lambda^{2}+\left(1+b_{2}\right) \lambda+1\right]
$$

It results that when $\delta=\left(b_{2}-1\right)\left(b_{2}+3\right)$ is positive, the two other eigenvalues of $A^{-1} B$ are real leading to the instability of the periodic solution. For $\delta<0, A^{-1} B$ has a complex conjugate pair of eigenvalues on the unit circle.

5.2 Forced System. A periodic solution of period $2 \pi / \omega$ is obtained for initial conditions $z_{00}=\left(\begin{array}{c}1 \\ y_{0}\end{array}\right), \dot{z}_{00}=\left(\begin{array}{c}u_{0} \\ 0\end{array}\right)$, and phase angle $\varphi_{0}=0,\left(y_{0}, u_{0}\right)$ defined by $(34)$.

Let us consider a perturbed motion related to initial conditions (47) and phase angle $\varphi=\varphi_{0}+d \varphi$. The corresponding free motion performed by the system for $t>0$, is obtained from (29), with $\dot{z}_{c}$ $=E \dot{z}_{0}$. This motion ends at $t=2 \pi / \omega+d T$, when $z_{1}(2 \pi / \omega+d T)=1$ and $\dot{z}_{1}(2 \pi / \omega+d T)>0$. Let us denote by $z_{f}, \dot{z}_{f}$ the positions and the velocities of the system at this time. Assuming small perturbations of the initial conditions and of the phase angle:

$$
\begin{gathered}
d z_{f}=z_{f}-z_{00}=\Gamma_{1} d z_{0}+\Gamma_{2}\left(E d \dot{z}_{0}+R \omega d \varphi\right)+n_{1} d T \\
d \dot{z}_{f}=\dot{z}_{f}-\dot{z}_{00}=\Gamma_{3} d z_{0}+\Gamma_{1}\left(E d \dot{z}_{0}+R \omega d \varphi\right)-R \omega d \varphi^{\prime}+n_{2} d T \\
d \varphi^{\prime}=\omega d T+d \varphi \\
n_{1}=\dot{z}_{00}=\left(\begin{array}{c}
u_{0} \\
0
\end{array}\right), \quad n_{2}=\Gamma_{2}^{-1}\left(\Gamma_{1}+I\right) n_{1}, \quad \Gamma_{j}=\Gamma_{j}(2 \pi / \omega) \quad(j=1,2,3)
\end{gathered}
$$

From (52), after the elimination of $d T$, we deduce the matrix $N_{0}$ giving the linear correspondence between the initial perturbations
$Y=(y, u, w, d \varphi)^{t}$ and the final ones $Y_{f}=\left(y_{f}, u_{f}, w_{f}, d \varphi^{\prime}\right)^{t}$ :

$$
\begin{gathered}
Y_{f}=N_{0} Y, \quad N_{0}=\tilde{A}^{-1} \tilde{B} \\
\tilde{A}=\left(\begin{array}{cccc}
1 & 0 & 0 & 0 \\
C_{12} & -C_{13} & -C_{14} & -\omega l_{1} \\
C_{22} & -C_{23} & -C_{24} & -\omega l_{2} \\
0 & 0 & 0 & 1
\end{array}\right), \\
\tilde{B}=\left(\begin{array}{cccc}
C_{22} & -C_{23} & C_{24} & \omega l_{2} \\
C_{12} & -C_{13} & C_{14} & \omega l_{1} \\
1 & 0 & 0 & 0 \\
-\chi C_{12} & \chi C_{13} & -\chi C_{14} & 1-\omega \chi l_{1}
\end{array}\right) \\
l_{1}=C_{13} R_{1}+C_{14} R_{2}, \quad l_{2}=C_{23} R_{1}+C_{24} R_{2}, \quad \chi=\frac{\omega}{u_{0}}
\end{gathered}
$$

Let us introduce the characteristic polynomial of the matrix $N_{0}$ :

$$
\widetilde{P}(\lambda) \equiv \operatorname{det}\left(N_{0}-\lambda I_{4}\right)=\lambda^{4}+a_{3} \lambda^{3}+a_{2} \lambda^{2}+a_{1} \lambda+a_{0}
$$

From the property $\operatorname{det}(\widetilde{A})=\operatorname{det}(\widetilde{B})=\operatorname{det}\left(\Gamma_{2}\right)$,we deduce

$$
a_{0}=\widetilde{P}(0)=\operatorname{det}\left(\widetilde{A}^{-1} \widetilde{B}\right)=1
$$

In this case it is impossible that all the eigenvalues of the matrix $N_{0}$ lie strictly inside the unit circle. The periodic solution is unstable except if all these eigenvalues lie on the unit circle. Moreover, for any $\lambda$ (see Appendix B) $\operatorname{det}(\tilde{A}-\lambda \widetilde{B}) \equiv \operatorname{det}(\widetilde{B}-\lambda \widetilde{A})$.

It results that $\widetilde{P}(\lambda) \equiv \lambda^{4} \widetilde{P}(1 / \lambda)$ and hence $a_{1}=a_{3}$. The conditions under which all the eigenvalues of $N_{0}$ lie on the unit circle are (Appendix B):

$$
\begin{gathered}
\left|a_{3}\right| \leqslant 4, \quad a_{2}-2 a_{3}+2 \geqslant 0, \quad a_{2}+2 a_{3}+2 \geqslant 0, \\
a_{3}^{2}-4 a_{2}+8 \geqslant 0
\end{gathered}
$$

In the more general case of a restitution coefficient $r(0<r<1)$, the stability conditions of periodic solutions have been obtained in Ref. [10].

\section{Stability of Periodic Motions (Soft Impact)}

6.1 Unforced System. When the stiffness of the obstacle is bounded and when there is no external excitation, the mathematical model of the system is given by (12) for the free motion and (13) for the constraint motion.

Let us consider a periodic motion of period $\tau_{0}+T_{0}=2 \pi / \omega$, where $\omega$ is an arbitrary positive value in this case. This periodic solution is related to the initial conditions $z_{00}=\left(\begin{array}{c}1 \\ y_{0}\end{array}\right), \dot{z}_{00}=\left(\begin{array}{l}u_{0} \\ w_{0}\end{array}\right)$, where $\left(y_{0}, u_{0}, w_{0}, \tau_{0}, T_{0}\right)$ are defined in terms of $\omega$ by (28) and the condition $\tau_{0}+T_{0}=2 \pi / \omega$.

Let us consider the perturbed motion defined by a set of new initial conditions (47).

This motion is defined in two steps:

- For $0 \leqslant t \leqslant \tau=\tau_{0}+d \tau$, the system performs a constraint motion ending when $z_{1}(\tau)=1$ and $\dot{z}_{1}(\tau)<0$. Let us denote by

$$
\begin{gathered}
z_{c}=z_{00}+d z_{c}, \quad \dot{z}_{c}=-\dot{z}_{00}+d \dot{z}_{c}, \\
d z_{c}=\left(\begin{array}{c}
0 \\
y_{c}
\end{array}\right), \quad d \dot{z}_{c}=\left(\begin{array}{c}
u_{c} \\
w_{c}
\end{array}\right)
\end{gathered}
$$

the positions and the velocities reached by the system at this time. 
- For $\tau \leqslant t \leqslant 2 \pi / \omega+d \theta$, the system performs a free motion finishing for $z_{1}(2 \pi / \omega+d \theta)=1, \dot{z}_{1}(2 \pi / \omega+d \theta)>0$. Let us denote by $z_{f}=z_{00}+d z_{f}, \dot{z}_{f}=\dot{z}_{00}+d \dot{z}_{f}$ the positions and the velocities reached by the system at this time.

Assuming small perturbations $d z_{0}, d \dot{z}_{0}$ of the initial conditions,

$$
\begin{aligned}
& d z_{c}=H_{1} d z_{0}+H_{2} d \dot{z}_{0}+p_{1} d \tau \\
& d \dot{z}_{c}=H_{3} d z_{0}+H_{1} d \dot{z}_{0}+p_{2} d \tau
\end{aligned}\left(H_{i}=H_{i}\left(\tau_{0}\right), i=1,2,3\right)
$$

$p_{1}=\dot{H}_{1}\left(z_{00}-d_{0}\right)+\dot{H}_{2} \dot{z}_{00}=-\dot{z}_{00}$

$p_{2}=\dot{H}_{3}\left(z_{00}-d_{0}\right)+\dot{H}_{1} \dot{z}_{00}=-H_{2}^{-1}\left(H_{1}+I\right) \dot{z}_{00}$

$\left(\dot{H}_{i}=\dot{H}_{i}\left(\tau_{0}\right), i=1,2,3\right)$

In a same way

$$
\begin{gathered}
d z_{f}=\Gamma_{1} d z_{c}+\Gamma_{2} d \dot{z}_{c}+p_{3} d \theta, \quad p_{3}=\dot{\Gamma}_{1} z_{00}-\dot{\Gamma}_{2} \dot{z}_{00}=\dot{z}_{00} \\
d \dot{z}_{f}=\Gamma_{3} d z_{c}+\Gamma_{1} d \dot{z}_{c}+p_{4} d \theta, \quad p_{4}=\dot{\Gamma}_{3} z_{00}-\dot{\Gamma}_{1} \dot{z}_{00}=\Gamma_{2}^{-1}\left(\Gamma_{1}+I\right) \dot{z}_{00} \\
\left(\Gamma_{i}=\Gamma_{i}\left(T_{0}\right), \dot{\Gamma}_{i}=\dot{\Gamma}_{i}\left(T_{0}\right), i=1,2,3\right)
\end{gathered}
$$

From (57) and (59), after the elimination of $d \tau$ and $d \theta$, we deduce the correspondence between the initial perturbations $(y u w)^{t}$ and the final ones $\left(y_{f} u_{f} w_{f}\right)^{t}$ :

$$
\begin{gathered}
\left(\begin{array}{l}
y_{f} \\
u_{f} \\
w_{f}
\end{array}\right)=A_{3}\left(\begin{array}{l}
y \\
u \\
w
\end{array}\right), \quad A_{3}=\bar{A}_{2} \bar{A}_{1}, \quad \bar{A}_{i}=M_{i}^{-1} N_{i}, \quad(i=1,2) \\
M_{1}=\left(\begin{array}{ccc}
1 & 0 & 0 \\
H_{12} & -H_{13} & -H_{14} \\
H_{22} & -H_{23} & -H_{24}
\end{array}\right), \\
N_{1}=\left(\begin{array}{lll}
H_{22}-\tilde{\chi} H_{12} & H_{23}-\tilde{\chi} H_{13} & H_{24}-\tilde{\chi} H_{14} \\
H_{12} & H_{13} & H_{14} \\
1+\tilde{\chi} H_{12} & \tilde{\chi} H_{13} & \tilde{\chi} H_{14}
\end{array}\right), \quad \tilde{\chi}=\frac{w_{0}}{u_{0}}
\end{gathered}
$$

$M_{2}$ and $N_{2}$ are deduced, respectively, from the expression of $M_{1}$ and $N_{1}$ by substituting the terms $H_{i j}$ by the terms $C_{i j}$. Let us introduce the characteristic polynomial $\bar{P}(\lambda)$ of the matrix $A_{3}$. From the property:

$$
\operatorname{det}\left(M_{i}\right)=-\operatorname{det}\left(N_{i}\right), \quad(i=1,2)
$$

we deduce that $\bar{P}(0)=\operatorname{det}\left(A_{3}\right)=1$. It is not possible in this case that all the eigenvalues of $A_{3}$ lie strictly inside the unit circle.

6.2 Forced System. Let us consider a periodic solution of system (35), of period $\tau_{0}+T_{0}=2 \pi / \omega$, with $\varphi=-\widetilde{\varphi}_{0}=-\omega \tau_{0} / 2$, related to the initial conditions $z_{00}=\left(\begin{array}{c}1 \\ y_{0}\end{array}\right), \quad \dot{z}_{00}=\left(\begin{array}{c}u_{0} \\ w_{0}\end{array}\right)$ where $\left(\tau_{0}, y_{0}, u_{0}, w_{0}\right)$ are deduced from the system (45).

The stability of this periodic solution is investigated by considering the motion related to the new initial conditions (47) and new phase angle $\varphi=-\omega \tau_{0} / 2+d \varphi$.

This motion is defined in two steps:

- For $0 \leqslant t \leqslant \tau=\tau_{0}+d \tau: z, \dot{z}$ are defined by (36). This motion ends when $z_{1}(\tau)=1$ and $\dot{z}_{1}(\tau)<0$. The positions and the velocities reached by the system at this final time are defined by (56).

- For $\tau \leqslant t \leqslant 2 \pi / \omega+d \theta$, the motion is defined by (38). This motion ends when $z_{1}(2 \pi / \omega+d \theta)=1, \dot{z}_{1}(2 \pi / \omega+d \theta)>0$. Let us denote by $z_{f}=z_{00}+d z_{f}, \dot{z}_{f}=\dot{z}_{00}+d \dot{z}_{f}$, the positions and the velocities reached by the system at this time.

Assuming small perturbations $d z_{0}, d \dot{z}_{0}$ of the initial conditions,

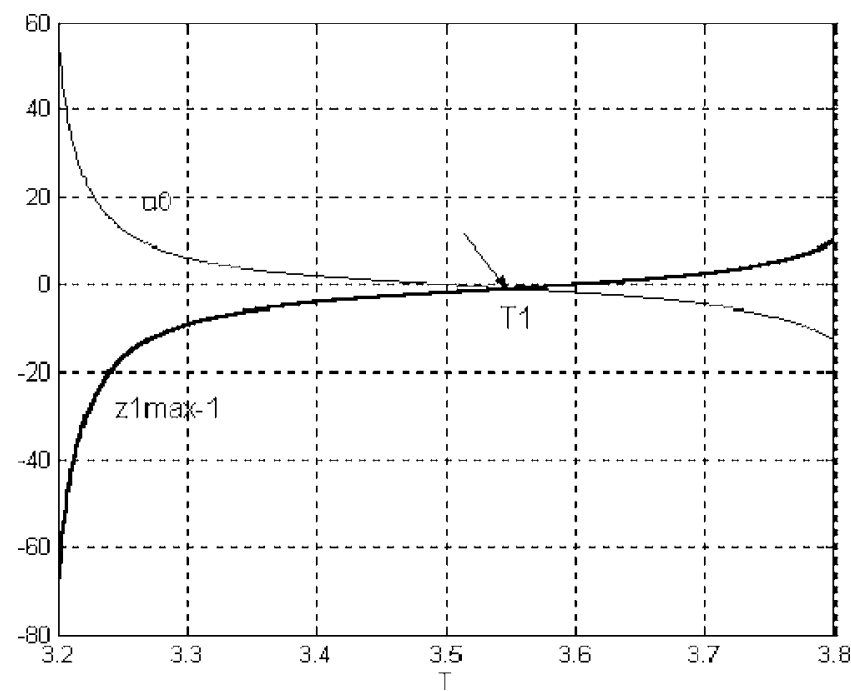

Fig. 2 Actual periodic impact solution (rigid impact, unforced system)

$$
\begin{aligned}
& d z_{c}=H_{1} d z_{0}+H_{2} d \dot{z}_{0}+\widetilde{p}_{1} d \tau+q_{1} d \varphi \\
& d \dot{z}_{c}=H_{3} d z_{0}+H_{1} d \dot{z}_{0}+\widetilde{p}_{2} d \tau+q_{2} d \varphi
\end{aligned}\left(H_{i}=H_{i}\left(\tau_{0}\right), i=1,2,3\right)
$$

$$
\begin{gathered}
\tilde{p}_{1}=-\dot{z}_{00}, \quad \tilde{p}_{2}=-H_{2}^{-1}\left(H_{1}+I\right)\left(\dot{z}_{00}-Q \omega \sin \widetilde{\varphi}_{0}\right)-Q \omega^{2} \cos \widetilde{\varphi}_{0} \\
q_{1}=-\left(H_{1}+I\right) Q \sin \widetilde{\varphi}_{0}+H_{2} Q \omega \cos \widetilde{\varphi}_{0}\left(q_{1}=\left(q_{11}, q_{12}\right)^{t}\right) \\
q_{2}=H_{2}^{-1}\left(H_{1}-I\right) q_{1}
\end{gathered}
$$

In a same way

$$
\begin{aligned}
& d z_{f}=\Gamma_{1} d z_{c}+\Gamma_{2} d \dot{z}_{c}+\widetilde{p}_{3} d T+q_{3} d \varphi^{\prime} \\
& d \dot{z}_{f}=\Gamma_{3} d z_{c}+\Gamma_{1} d \dot{z}_{c}+\widetilde{p}_{4} d T+q_{4} d \varphi^{\prime}
\end{aligned}\left(\Gamma_{i}=\Gamma_{i}\left(T_{0}\right), i=1,2,3\right)
$$

$$
\begin{gathered}
d T=d \theta-d \tau, \quad d \varphi^{\prime}=\omega d \theta+d \varphi \\
q_{3}=\left(\Gamma_{1}+I\right) R \sin \widetilde{\varphi}_{0}+\Gamma_{2} R \omega \cos \widetilde{\varphi}_{0}\left(q_{3}=\left(q_{31}, q_{32}\right)^{t}\right) \\
q_{4}=\Gamma_{2}^{-1}\left(\Gamma_{1}-I\right) q_{3} \\
\tilde{p}_{3}=\dot{z}_{00}-\omega q_{3} \\
\tilde{p}_{4}=\Gamma_{2}^{-1}\left(\Gamma_{1}+I\right)\left(\dot{z}_{00}-R \omega \sin \widetilde{\varphi}_{0}\right)-\omega \Gamma_{2}^{-1}\left(\Gamma_{1}-I\right) q_{3}-R \omega^{2} \cos \widetilde{\varphi}_{0} \\
\left(\tilde{p}_{3}=\left(\tilde{p}_{31}, \tilde{p}_{32}\right)^{t}\right)
\end{gathered}
$$

From (63) and (64), after the elimination of $d \tau$ and $d T$, we deduce the matrix $A_{3}$ (see Appendix C) giving the correspondence between the initial perturbations and the final ones:

$$
\left(\begin{array}{c}
y_{f} \\
u_{f} \\
w_{f} \\
d \varphi^{\prime}
\end{array}\right)=\widetilde{A}_{3}\left(\begin{array}{c}
y \\
u \\
w \\
d \varphi
\end{array}\right) \quad \tilde{A}_{3}=\left(\begin{array}{cc}
\tilde{A}_{2} & \tilde{N}_{2} \\
\tilde{M}_{3} & \tilde{N}_{3}
\end{array}\right)
$$

The stability of the periodic motion is determined by the eigenvalues of the matrix $\widetilde{A}_{3}$.

\section{Numerical Results}

Some numerical investigations are performed for the following values of the parameters: $k_{1}=k_{3}=1, k_{2}=5, m_{1}=1, m_{2}=2, P_{1}$ $=2 / 3, P_{2}=0$. The corresponding eigenvalues of the free system are: $\omega_{1}=1.7958, \omega_{2}=0.8805$, while the eigenvalues of the constraint system are: $\sigma_{1}=1.8347, \sigma_{2}=1.2783$. An example of peri- 


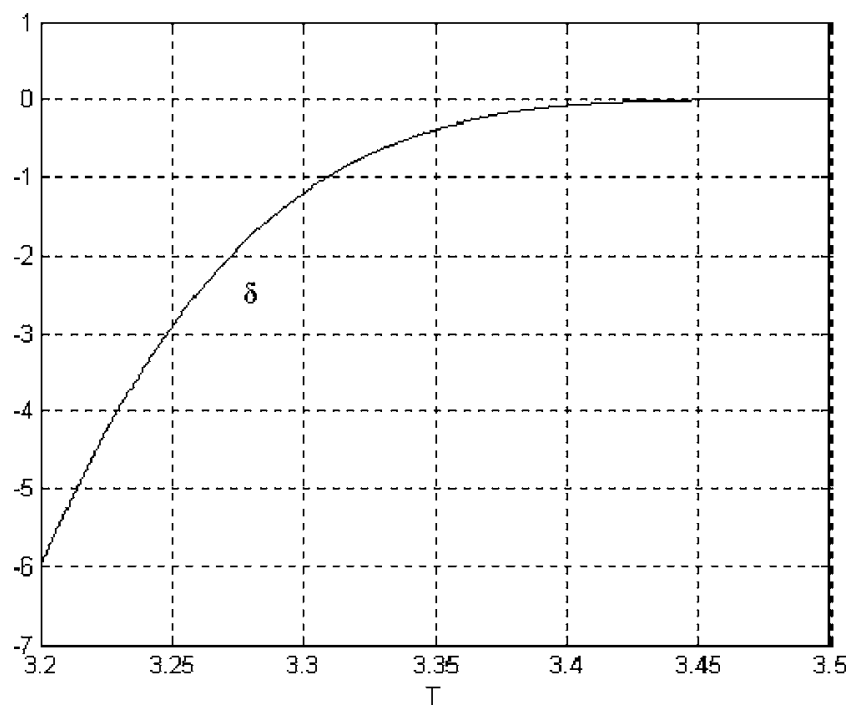

Fig. 3 Stability of the periodic solution (rigid impact, unforced system)

odic solutions obtained for forced and unforced systems is shown in Ref. [10] in the rigid impact and soft impact cases. Stability conditions are investigated in the case of rigid impact, for both unforced and forced systems. In both cases, the initial conditions (10) (unforced system) and (34) (forced system) are related to an actual periodic solution of period $T$ only if $u_{0}>0, z_{1}(t)<1,0$ $<t<T$. The initial velocity of the impacting mass is reformulated as:

$$
\begin{gathered}
u_{0 \mathrm{NF}}=\frac{\left(\lambda_{1}-\lambda_{2}\right) x_{1} x_{2}}{\tilde{D}}, \quad \tilde{D}=\lambda_{2} x_{2} y_{1}-\lambda_{1} x_{1} y_{2} \\
(\text { unforced system) } \\
x_{i}=\omega_{i} \sin \left(\omega_{i} T / 2\right), \quad y_{i}=\cos \left(\omega_{i} T / 2\right) \\
u_{0 \mathrm{~F}}=\left(1-R_{1}\right) u_{0 \mathrm{NF}}, \quad T=2 \pi / \omega \text { (forced system) }
\end{gathered}
$$

In both systems, limiting cases are obtained for $x_{i}=0,(i=1,2)$ or $T_{i}=2 k \pi / \omega_{i},(k=1,2, \ldots)$. On the other hand, $u_{0 \mathrm{NF}}$ and $u_{0 \mathrm{~F}}$ are not defined if $\widetilde{D}=0$. The corresponding motion of the impacting mass is given by:

$$
\begin{aligned}
& z_{1 \mathrm{NF}}(t) \\
& \quad=\frac{\lambda_{2} x_{2} \cos \omega_{1}(t-T / 2)-\lambda_{1} x_{1} \cos \omega_{2}(t-T / 2)}{\widetilde{D}} \text { (unforced system) } \\
& z_{1 \mathrm{~F}}(t)=R_{1} \cos \omega t+\left(1-R_{1}\right) z_{1 \mathrm{NF}}(t), \quad T=2 \pi / \omega \text { (forced system) }
\end{aligned}
$$

The motion of the impacting mass is symmetrical with respect to $T / 2(\pi / \omega$ for the forced system $)$ and the maximum value of $z_{1}(t)$ is obtained at this point. For the unforced system, the behavior of $u_{0}, z_{1} \max -1$ and $\delta$ (stability condition) are investigated in terms of the period $T$. Figure 2 is related to the interval $[3.2,3.8]$ which contains the value $T_{1}=2 \pi / \omega_{1} \simeq 3.4989$ for which $u_{0}=0$. Figure 3 shows the behavior of $\delta$ for $3.2<T<T_{1}$. Figure 4 is a zoom of the last figure in the interval $\left[3.49, T_{1}\right]$ which includes a bifurcation value $T_{1}^{\prime} \simeq 3.4911$ of the period for which a change of the stability occurs. (For this value, two eigenvalues of the matrix $A^{-1} B$ are equal to -1 .) For the forced system, the behavior of $u_{0}, z_{1} \max -1$ (Fig. 5) and the stability conditions (Fig. 6) depends on the value of $\omega$ or equivalently on the value of $T=2 \pi / \omega$. Figure 5 is related

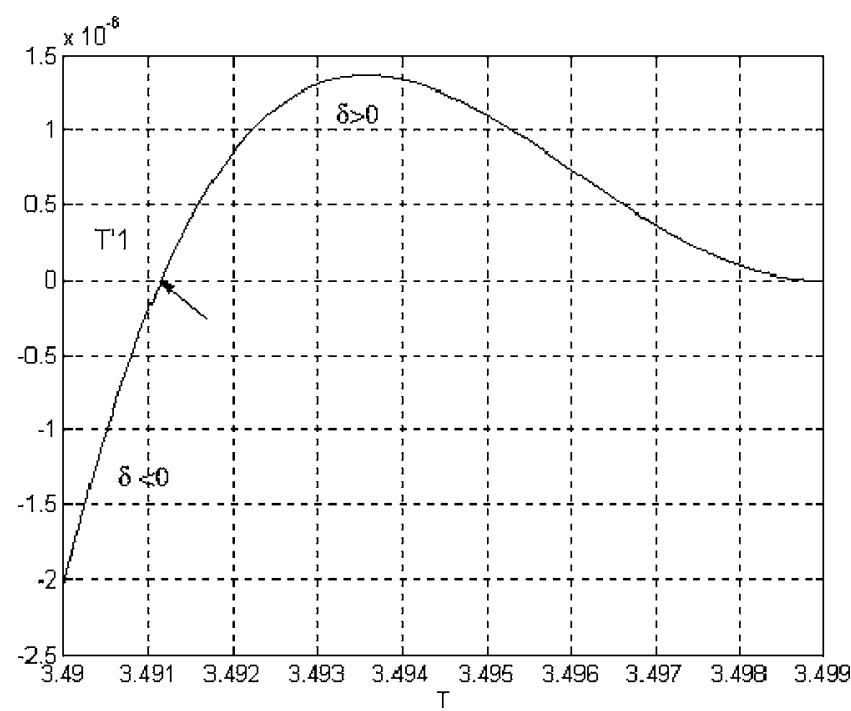

Fig. 4 Bifurcation value of the period (rigid impact, unforced system)

to the interval $5<T<6(1.0472<\omega<1.2566)$ while in Fig. 6 , the functions

$$
\begin{aligned}
n_{1}^{\prime}= & 16-a_{3}^{2}, \quad n_{2}^{\prime}=a_{2}-2 a_{3}+2, \\
& n_{3}^{\prime}=a_{2}+2 a_{3}+2, \quad n_{4}^{\prime}=a_{3}^{2}-4 a_{2}+8
\end{aligned}
$$

occurring in the stability conditions (55) are plotted in this interval.

Three bifurcation values $\left(T_{2}^{\prime} \simeq 5.0809, T_{3}^{\prime} \simeq 5.2002\right.$, and $T_{4}^{\prime}$ $\simeq 5.8109$ ) appear in this interval: The two first values are related to the case of two eigenvalues of the matrix $N_{0}$ equal to -1 while the last value is related to the case of complex eigenvalues $\tilde{\lambda_{k}}$ $(k=1, \ldots, 4)$ on the unit circle, with $\tilde{\lambda}_{1}=\tilde{\lambda_{2}}=1 / \tilde{\lambda}_{3}=1 / \tilde{\lambda}_{4}$. Stability and bifurcation conditions in the case of soft impact will be the subject of further numerical investigations.

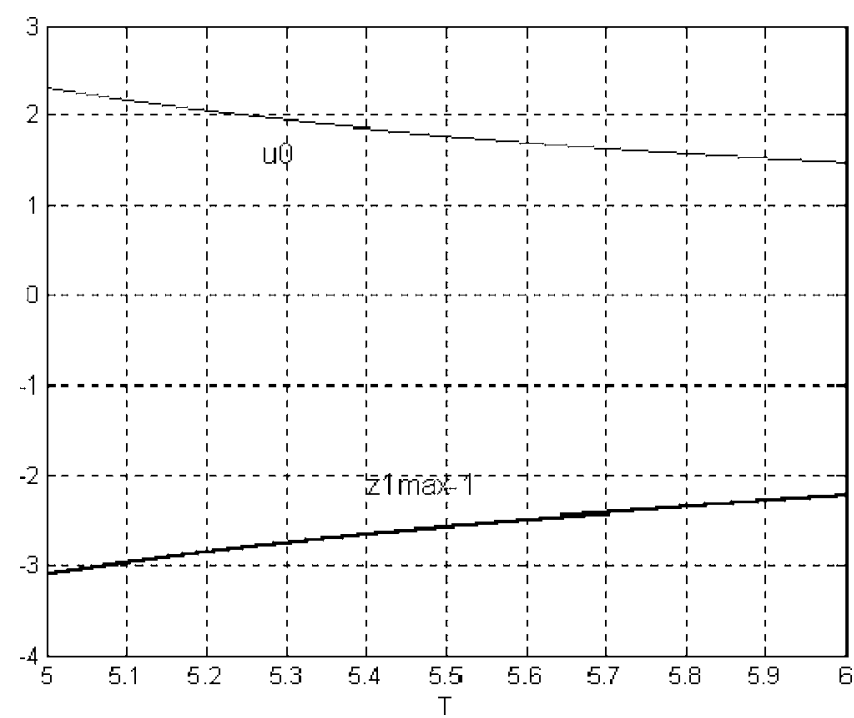

Fig. 5 Actual periodic solution (rigid impact, forced system) 


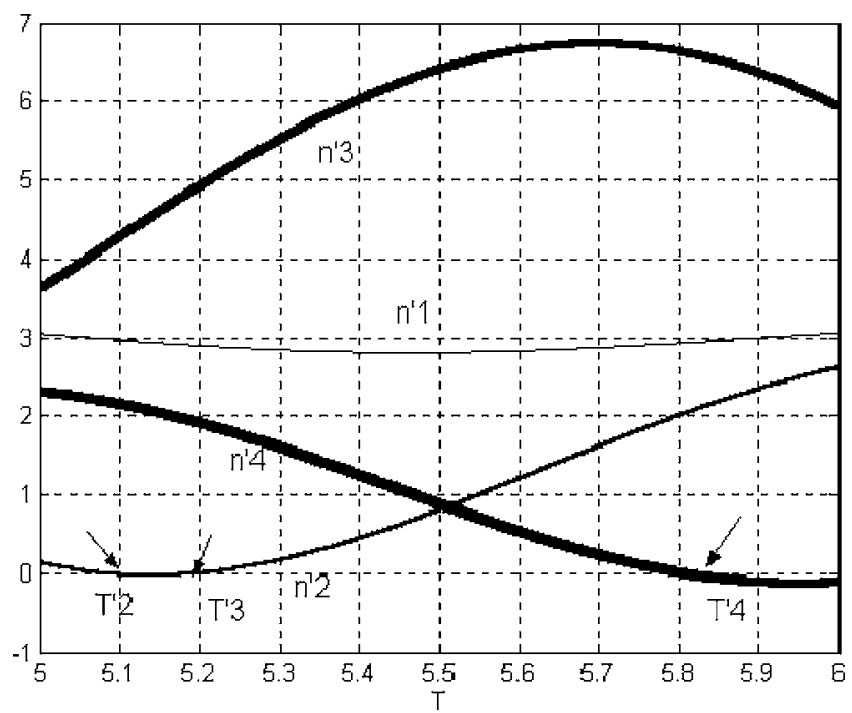

Fig. 6 Bifurcation values of the period $T=2 \pi / \omega$ (rigid impact, forced system)

\section{Appendix A: Discussion About the Existence of Periodic Motions (Soft Impact, Unforced System)}

The second solution arising from conditions (25) is defined by:

$$
\begin{gathered}
P X_{1}=0, \quad \operatorname{det}(P)=0, \quad X_{1}=X_{2}, \quad z_{1 c}=1 \\
P=\left(P_{i j}\right)=\left[H_{2}^{-1}\left(H_{1}+I\right)+\Gamma_{2}^{-1}\left(\Gamma_{1}+I\right)\right]
\end{gathered}
$$

From $P X_{1}=0$ and $X_{1}=\left(\begin{array}{l}X_{11} \\ X_{12}\end{array}\right)$ where $X_{11}=0$ and $X_{12} \neq 0$, we deduce:

$$
P_{12}=0, \quad P_{22}=0
$$

These two equations give discrete values of $\tau$ and $\mathrm{T}$ independent of the initial conditions. $y, u, w$ are defined by three scalar equations deduced from the conditions:

$$
\begin{gathered}
X_{1}=X_{2}, \quad X_{21}=0 \\
X_{2}=\left(\begin{array}{l}
X_{21} \\
X_{22}
\end{array}\right)
\end{gathered}
$$

Let us introduce

$$
P_{1}=\left(\begin{array}{ll}
\alpha_{1} & a \\
\alpha_{2} & b
\end{array}\right), \quad P_{2}=\left(\begin{array}{ll}
\beta_{1} & a \\
\beta_{2} & b
\end{array}\right)
$$

where $\left(\alpha_{1}, \alpha_{2}, \beta_{1}, \beta_{2}, a, b\right)$ are deduced from the definition (23) of $P_{1}$ and $P_{2}$ and the property (A2). From (A3), we deduce

$$
\begin{gathered}
\left(H_{1}-\Gamma_{1}\right) z_{0}+\left(H_{2}+\Gamma_{2}\right) \dot{z}_{0}=\left(H_{1}-I\right) d_{0} \\
-y C_{12}+u C_{13}+w C_{14}=C_{11}+1
\end{gathered}
$$

From $H_{1}+I=H_{2} P_{1}, \Gamma_{1}-I=\Gamma_{2} P_{2}$, (A5) gives:

$$
\begin{gathered}
H_{2} \xi+\Gamma_{2} \eta=\left(H_{1}-I\right) d_{0} \\
\xi=P_{1} z_{0}+\dot{z}_{0}=\left(\begin{array}{c}
\alpha_{1}+p \\
\alpha_{2}+q
\end{array}\right), \\
\eta=P_{2} z_{0}+\dot{z}_{0}=\left(\begin{array}{c}
\beta_{1}+p \\
\beta_{2}+q
\end{array}\right), \quad \begin{array}{l}
p=u+a y \\
q=w+b y
\end{array}
\end{gathered}
$$

From (A7) it is possible to deduce $Q=\left(\begin{array}{c}p \\ q\end{array}\right)$ :

$$
\begin{gathered}
Q=\left(H_{2}+\Gamma_{2}\right)^{-1}\left[\left(H_{1}-I\right) d_{0}-H_{2} \xi_{0}-\Gamma_{2} \eta_{0}\right], \quad \xi_{0}=\left(\begin{array}{c}
\alpha_{1} \\
\alpha_{2}
\end{array}\right), \\
\eta_{0}=\left(\begin{array}{c}
\beta_{1} \\
\beta_{2}
\end{array}\right)
\end{gathered}
$$

The condition (A6) can also be expressed in terms of $p$ and $q$ :

$$
C_{13} p+C_{14} q=C_{11}-1
$$

Equations (A8) and (A9) provide 3 scalar equations for the determination of the two parameters $p$ and $q$. The condition of compatibility for this system is given by:

$$
\begin{gathered}
\operatorname{det}\left(\begin{array}{lll}
C_{13} & C_{14} & C_{11}-1 \\
H_{13} & H_{14} & \tilde{L}_{1} \\
H_{23}+C_{23} & H_{24}+C_{24} & \tilde{L}_{2}
\end{array}\right)=0 \\
\tilde{L}_{1}=d_{2} H_{12}+\left(1-H_{11}\right)\left(1-d_{1}\right) \\
\tilde{L}_{2}=\left(d_{1}-1\right) H_{21}+d_{2}\left(H_{22}-1\right)+C_{21}
\end{gathered}
$$

This compatibility condition is not fulfilled in the general case and the problem has no solution.

\section{Appendix B: Properties of the $N_{o}$ Matrix (Rigid Impact, Forced System)}

$$
\begin{aligned}
& \operatorname{det}(\widetilde{B}-\lambda \widetilde{A}) \\
& =\left|\begin{array}{cccc}
C_{22}-\lambda & -C_{23} & C_{24} & \omega l_{2} \\
C_{12}(1-\lambda) & C_{13}(\lambda-1) & C_{14}(\lambda+1) & \omega l_{1}(\lambda+1) \\
1-\lambda C_{22} & \lambda C_{23} & \lambda C_{24} & \omega l_{2} \lambda \\
-\tilde{\chi} C_{12} & \tilde{\chi} C_{13} & -\tilde{\chi} C_{14} & 1-\lambda-\omega l_{1} \tilde{\chi}
\end{array}\right|(\mathrm{B} 1)
\end{aligned}
$$

$\operatorname{det}(\widetilde{A}-\lambda \widetilde{B})$

$$
=\left|\begin{array}{cccc}
1-\lambda C_{22} & \lambda C_{23} & -\lambda C_{24} & -\lambda \omega l_{2} \\
C_{12}(1-\lambda) & C_{13}(\lambda-1) & -C_{14}(\lambda+1) & -\omega l_{1}(\lambda+1) \\
-\lambda+C_{22} & -C_{23} & -C_{24} & -\omega l_{2} \\
\lambda \tilde{\chi} C_{12} & -\lambda \tilde{\chi} C_{13} & \lambda \tilde{\chi} C_{14} & 1-\lambda+\omega l_{1} \tilde{\chi} \lambda
\end{array}\right|
$$

or,

$$
\begin{aligned}
& \operatorname{det}(\tilde{A}-\lambda \widetilde{B}) \\
& =\left|\begin{array}{cccc}
1-\lambda C_{22} & \lambda C_{23} & -\lambda C_{24} & -\lambda \omega l_{2} \\
C_{12}(1-\lambda) & C_{13}(\lambda-1) & -C_{14}(\lambda+1) & -\omega l_{1}(\lambda+1) \\
-\lambda+C_{22} & -C_{23} & -C_{24} & -\omega l_{2} \\
\tilde{\chi} C_{12} & -\tilde{\chi} C_{13} & -\tilde{\chi} C_{14} & 1-\lambda+\omega l_{1} \tilde{\chi}
\end{array}\right|
\end{aligned}
$$

It is not difficult to show, after some permutations of rows and columns in (B3), that $\operatorname{det}(\widetilde{A}-\lambda \widetilde{B}) \equiv \operatorname{det}(\widetilde{B}-\lambda \widetilde{A})$. It results that $\widetilde{P}(\lambda) \equiv \lambda^{2} Q(\widetilde{\sigma}), Q(\widetilde{\sigma}) \equiv \widetilde{\sigma}^{2}+a_{3} \widetilde{\sigma}+a_{2}-2, \quad \widetilde{\sigma}=\lambda+1 / \lambda$. Let us assume that the roots $\tilde{\lambda_{k}}(\mathrm{k}=1, \ldots, 4)$ of $\widetilde{P}(\lambda)$ are on the unit circle: $\tilde{\lambda_{1}}=e^{i \theta_{1}}, \tilde{\lambda_{2}}=e^{i \theta_{2}}, \tilde{\lambda_{3}}=1 / \tilde{\lambda_{1}}, \tilde{\lambda_{4}}=1 / \tilde{\lambda_{2}}$. The roots of $Q(\widetilde{\sigma})$ in this case are $\widetilde{\sigma}_{j}=2 \cos \theta_{j},(j=1,2)$. We deduce that all the eigenvalues of the matrix $N_{0}$ are on the unit circle if the roots of $Q(\widetilde{\sigma})$ are real and between -2 and 2. From this, we deduce the conditions (55).

\section{Appendix C: Obtention of the $\widetilde{A}_{3}$ Matrix (Soft Impact, Forced System)}

$$
\begin{gathered}
\tilde{A}_{2}=\left(\widetilde{C}-\bar{p} \bar{M}_{2}\right) \tilde{A}_{1}+\left(\omega \widetilde{q} \widetilde{\chi}_{2}-\bar{\chi}_{2} \widetilde{p}\right) \bar{M}_{1}, \\
\tilde{N}_{2}=\left(\widetilde{C}-\bar{p}_{2} \bar{M}_{2}\right) \tilde{N}_{1}+\widetilde{\chi}_{1} \widetilde{\chi}_{2} \tilde{q}-\chi_{3} \tilde{p}
\end{gathered}
$$




$$
\begin{aligned}
& \tilde{M}_{3}=\omega\left(\bar{M}_{1}-\bar{M}_{2} \tilde{A}_{1}\right), \quad \tilde{N}_{3}=1+\omega\left(\chi_{1}-\chi_{3}-\bar{M}_{2} \tilde{N}_{1}\right) \\
& p=\left(\begin{array}{l}
-w_{0} \\
\widetilde{p}_{2}
\end{array}\right), \quad \tilde{p}=\left(\begin{array}{l}
\widetilde{p}_{32} \\
\widetilde{p}_{4}
\end{array}\right), \quad q=\left(\begin{array}{l}
q_{12} \\
q_{2}
\end{array}\right), \quad \widetilde{q}=\left(\begin{array}{l}
q_{32} \\
q_{4}
\end{array}\right), \quad \bar{p}=\widetilde{p}+\omega \tilde{q} \\
& \chi_{1}=\frac{q_{11}}{u_{0}}, \quad \tilde{\chi}_{1}=1+\omega \chi_{1}, \quad \chi_{2}=\tilde{p}_{31}+\omega q_{31}, \quad \bar{\chi}_{2}=\omega \frac{q_{31}}{\chi_{2}}, \\
& \tilde{\chi}_{2}=1-\bar{\chi}_{2}, \quad \chi_{3}=\frac{\bar{\chi}_{2} \tilde{\chi}_{1}}{\omega} \\
& \widetilde{H}=\left(\begin{array}{l}
H_{22} H_{23} H_{24} \\
H_{32} H_{11} H_{12} \\
H_{42} H_{21} H_{22}
\end{array}\right), \quad \widetilde{C}=\left(\begin{array}{l}
C_{22} C_{23} C_{24} \\
C_{32} C_{11} C_{12} \\
C_{42} C_{21} C_{22}
\end{array}\right), \\
& \bar{M}_{1}=\frac{1}{u_{0}}\left(H_{12} H_{13} H_{14}\right), \quad \bar{M}_{2}=\frac{1}{\chi_{2}}\left(C_{12} C_{13} C_{14}\right) \\
& \widetilde{A}_{1}=\widetilde{H}+p \bar{M}_{1}, \quad \tilde{N}_{1}=q+\chi_{1} p
\end{aligned}
$$

\section{References}

[1] Shaw, S. W., and Holmes, P. J., 1983, "A Periodically Forced Piecewise Linear Oscillator,” J. Sound Vib., 90(1), pp. 129-155.

[2] Shaw, S. W., and Holmes, P. J., 1983, "A Periodically Forced Impact Oscillator with Large Dissipation,” J. Appl. Mech., 50, pp. 849-857.

[3] Hindmarsh, M. B., and Jeffries, D. J., 1984, "On the Motions of the Impact Oscillator," J. Phys. A, 17, pp. 1791-1803.

[4] Peterka, F., 2001, "Dynamics of Oscillator with Soft Impacts," Proc. of the ASME 2001 Design Engineering Technical Conferences (CDROM), Pittsburgh, USA.

[5] Aidanpan, J. O, and Gupta, R. D., 1993, "Periodic and Chaotic Behavior of a Threshold-Limited Two Degree of Freedom System," J. Sound Vib., 165(2), pp. 305-327.

[6] Luo, G. W., and Xie, J. H, 1998, "Hopf Bifurcation of a Two Degree of Freedom Vibro-Impact System,” J. Sound Vib., 213(3), pp. 391-408.

[7] Valente, A. X., McClamroch, N. H., and Mezie, I., 2003, "Hybrid Impact of Two Coupled Oscillators that can Impact a Fixed Stop," Int. J. Non-Linear Mech., 38, pp. 677-689.

[8] Natsiavas, S., 1993, "Dynamics of Multiple-Degree-of-Freedom Oscillators with Colliding Components," J. Sound Vib., 165(3), pp. 439-453.

[9] Pascal, M., Stepanov, S., and Hassan, S., 2006, "An Analytical Investigation of the Periodic Motions of a Two Degree of Freedom Oscillator with Elastic Obstacles," J. Comput. Methods Sci. Eng. (to be published).

[10] Pascal, M., "Analytical Investigation of the Dynamics of a Non Linear Structure with Two Degrees of Freedom," submitted to ASME 2005 IDETC/CIE, September 2005, Long Beach, CA. 